\title{
On the Origin of Astrophysical Jets: a Review
}

\author{
J. H. Beall*† \\ Space Sciences Division, NRL \& St. John's College, USA \\ E-mail: beallesjc.edu
}

Astrophysical jets have been defined as linear structures detected in the sky, typically bi-polar, and originating from a common source. This review continues a series that considers our evolving understanding of the nature of astrophysical jets. Perhaps ironically, the original modeling consisted of analytically solveable estimates of radiation processes based on spherical symmetry due to the difficulties of analytical solutions without such simplifications.

We now know that jets are associated with outflows originating from accretion processes in starforming regions, compact objects (such as neutron stars and black holes), active galactic nuclei, and pulsars. In pulsars, the jet production can be drawn from angular momentum loss from the pulsar. In accreting systems, the jet production seems to be entangled intimately with processes in the accretion disks around the compact objects. The jets can be extremely powerful in terms both of their kinetic luminosity and radiative signatures, and often emit radiation over the entire electromagnetic spectrum, from radio to $\gamma$-rays. Astrophysical jets often appear to be one-sided or having asymmetric brightness in one of the "lobes." This phenomenon is usually interpreted as evidence of bulk relativistic motion of the emitting particles which produces a Doppler boosting of the approaching lobe's brightness, and a dimunition of the brightness of the jet lobe receeding from the observer.

In the last three decades and more and as a result of observing campaigns using detectors sensitive from radio to $\gamma$-ray energies, theoretical models of these sources have become richer and more complex. As noted above, models have moved from assumptions of isotropy that made analytic calculations possible, to fully anisotropic models of emission from the jets and their interactions with the interstellar and intra-cluster medium. Such anisotropic calculations are only possible because we now have extensive computational resources that can solve the rather complex emission models that result from such anisotropies. In addition, the degree of international cooperation required for observing campaigns of these sorts is remarkable.

Such observations have most recently allowed us to extimate the actual constitution of the astrophysical jets. Recent observation (see e.g., ICECUBE COLLABORATION (July 2018) by the neutrino detector system, Ice Cube, in concert with coordinated observations by MAGIC Collaboration Cherenkov telescopes and the Fermi LAT, have for the first time allowed us to give credence to the speculation that the jets consist of hadronic elements.

In this paper, I discuss some relevant observations from these efforts and the theoretical interpretations they have occasioned.

keywords: astrophysical jets, active galactic nuclei, UHE cosmic rays, quasars, microquasars

Frontier Research in Astrophysics - III (FRAPWS2018)

28 May - 2 June 2018

Mondello (Palermo), Italy

* Speaker.

$\dagger$ 


\section{Introduction}

Astrophysical jets have been defined as linear structures observed on the sky, sometimes bipolar, but sometimes seeming to be one-sided, and originating from a common source. Jets are typically associated with outflows originating from accretion processes in star-forming regions and in close binary systems containing compact objects (i.e., white dwarfs, neutron stars, and black holes), and of course in active galactic nuclei (AGNs), and galaxy clusters. They also appear to be present in $\gamma$-ray bursts associated with stellar collapse when the jet is pointed at the Earth, and in some pulsars. In pulsars, the required energy for jet production can be drawn from angular momentum loss of the pulsar. In accreting systems, the jet production seems to be entangled intimately with processes in the accretion disks around the compact objects.

Astrophysical jets often appear to be one-sided or having asymmetric brightness in one of the "lobes." This phenomenon is usually interpreted as evidence of bulk relativistic motion of the emitting particles which produces a Doppler boosting of the approaching lobe's brightness, and a dimunition of the brightness of the jet lobe receeding from the observer.

The jets can be extremely powerful in terms both of their kinetic luminosity and radiative signatures, and often emit radiation over the entire electromagnetic spectrum, from radio to $\gamma$-rays. In fact, Beall et al. (1978) have noted that the luninosity of the central source, which is clearly connected to the giant radio lobes via the jets flowing outward from the core of Cen A, could in fact provide the energy of the giant radio lobes.

The results of these campaigns show both similarities and significant differences in the data from some epochs of galactic microquasars, including GRS 1915+105 (Mirabel, et al., 1998; Rahoui et al., 2008), the concurrent radio and x-ray data (Beall et al., 1978) on Centaurus A (NGC 5128), 3C120 (Marscher (2006), and 3C454.3 as reported by Bonning et al. (2009), and Cygnus A (Carilli, et al., 1996), to name a few. The Bonning et al. analysis of the 3C454.3 data showed the first results from the Fermi LAT Space Telescope (Abdo 2009a, 2009b) for the concurrent variability at optical, UV, IR, and $\gamma$-ray frequencies for that source.

In combination with observations of microquasars and quasars from the MOJAVE Collaboration (see, e.g., Lister et al., 2009), these efforts provide an understanding of the time-dependent evolution of these sources at milliarcsecond resolutions (i.e., parsec for AGNs, and Astronomical Unit scales for microquasars).

The recent possibility of observational advances via millimeter wave interferometers (see, also, e.g., Beall 2015 for a review), have the potential of allowing us to observe the remarkable variability and structures on even finer scales.

Because of these campaigns both past and future, models of jet sources have progressed from earlier assumptions of isotropy that made analytic calculations possible, to fully anisotropic models of emission from the jets and their interactions with the interstellar and intra-cluster mediums. Furthermore, observations of the temporal behavior on milliarcsecond scales have allowed us to undertand that the source regions are not simple, but have a complex and evolving structure.

In large measure, such models of anisotropic structures and temporal variablility are only possible because we now have extensive computational resources to model the jets' structures. In addition, the degree of international cooperation required for observing campaigns of these sorts is remarkable, since the instruments include among others the Very Large Array (VLA), the Very 
Long Baseline Array (VLBA), and entire constellations of satellite instruments, often working in concert. In this paper, I discuss some relevant observations from these efforts and the theoretical interpretations they have occasioned.

In blazar sources (see, e.g., Ulrich et al. 1997, and Marscher, 2006) there seems to be a confirmed connection of jets with accretion disks. In sources without large-scale linear structures (i.e., jets), as Ulrich et al. (1997) note, the source variability could result from the complex interactions of the accretion disk with an x-ray emitting corona, and perhaps with the gravitational potential of the disks, themselves. But to the extent that "small" jets are present in these sources, the disk-jet interaction must still be of paramount importance, since it provides a mechanism for carrying away energy from the disk.

Current theories (see e.g., Hawley 2003 and Bisnovatyi-Kogan, et al., 2013 for a discussion of disk structure and jet-launch issues, respectively) suppose that the jet is formed and accelerated in the inner regions of the accretion disk. But even if this is true in all sources, it is still unclear whether or not astrophysical jets with shorter propagation lengths are essentially different in constitution from those that have much longer ranges, or whether the material through with the jet propagates determines the extent of the observational structures we call jets. At all events, the complexities of the jet-ambient medium interaction must have a great deal to do with the ultimate size of emitting region.

This sort of argument has applications to both quasars and microquasars, especially if essentially similar physical processes occurs in all these objects (see, e.g., Beall, 2003, and Marscher, 2006). To some, it has become plausible that essentially the same physics is working over a broad range of temporal, spatial, and luminosity scales. Hannikainen (2008) and Chaty $(1998,2007)$ have discussed some of the emission characteristics of microquasars, and Paredes (2007) has considered the role of microquasars and AGNs as sources of high energy $\gamma$-ray emission. In fact, the early reports of the concurrent radio and x-ray variability of Centaurus A can be plausibly interpreted as the launch of a jet from Cen A's central source into the complex structures in its core. Additionally, these observations are remarkably similar to the observations of galactic microquasars and AGNs, including the observations from the Fermi LAT Space Telescope of concurrent $\gamma$-ray, IR, optical, and UV variability of 3C454.3 (Bonning et al., 2009), and observations by Madejski et al. (1997) for BL Lac, among others.

\section{Jets from Accretion Disks in Star-Forming Regions}

There is evidence for accretion disks associated with the bipolar outflows in star-forming regions such as the rho Ophiuchus cloud (see, e.g., Beall 1987, and the rho Ophiuchus jet observations taken from K. Tachihara's Ph.D. thesis). The data therein interpreted show that the IR spectra in the centers of the regions of bi-polar flows are consistent the emission expected from accretion disks with the thermal gradient expected from a Shakura-Sunyaev (1973) accretion disk. The spectrum calculated by Beall (op. cit.) agrees with the illustration presented by Hannikainen (2005).

Recent observations bear out this hypothesis. For example, the observations of HL Tauri with the Atacama Large Millimeter/Sub-Millimeter Array (ALMA) presented in Figure 1 clearly show 
the resolved accretion disk structure of the protostellar system beginning to segregate itself into rings that are hypothesized to become proto-planets.

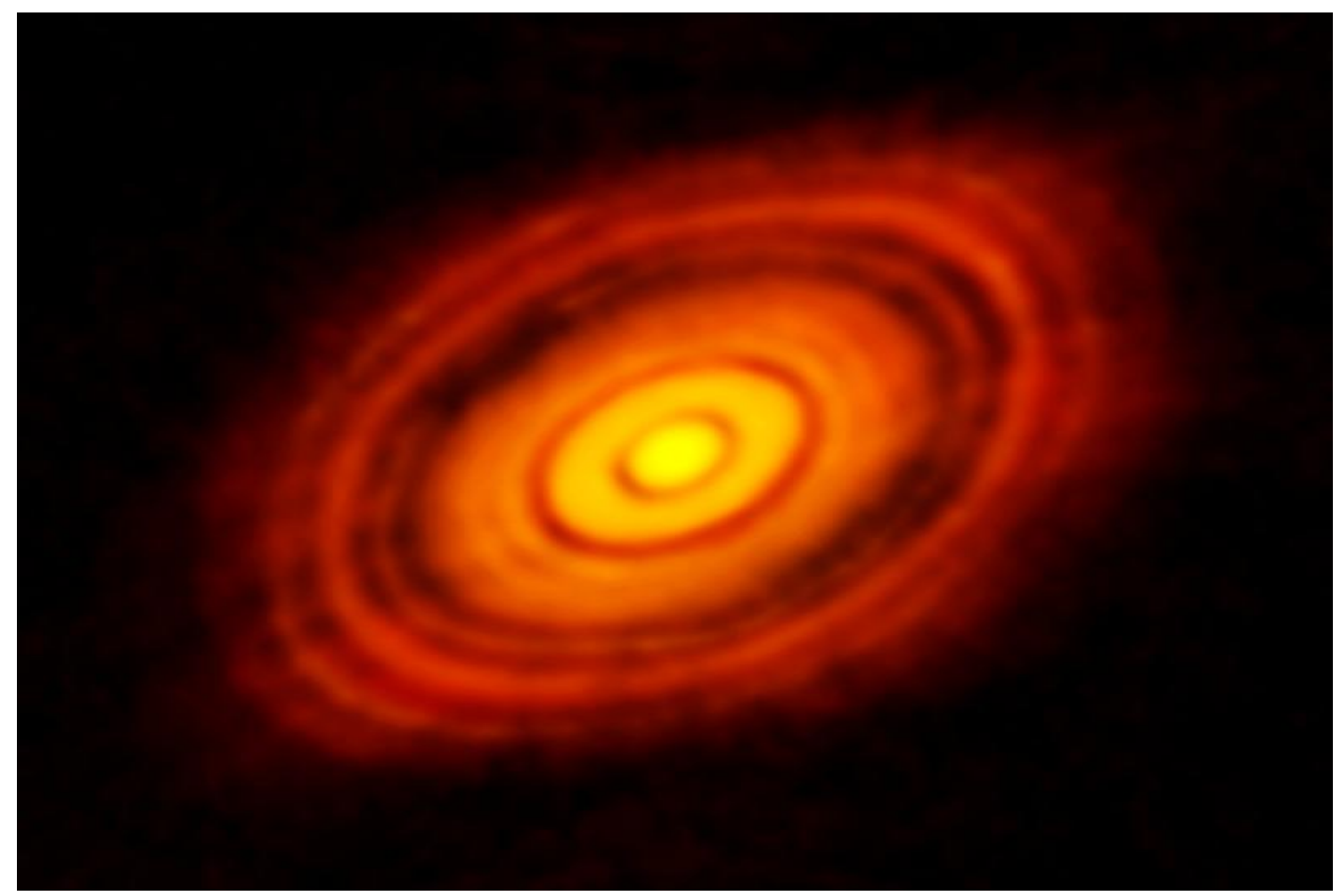

Figure 1: HL Tauri: note the ring-like structure that is likely to represent planetary formation in the protostellar system. This image is from ALMA (Atacama Large Millimeter/sub-millimeter Array (see, e.g., http://apod.nasa.gov/apod/ap141110.html). Radio polarization data from the CARMA (Combined Array for Research in Millimeter-wave Astronomy) observatory show magnetic structures consistent with a jet perpendicular to the disk plane.

Remarkably, this resolved accretion disk is clearly associated with a jet structure (Rodriguez, et al., 1994) for data taken by the VLA on arcsecond scales.

The observations from ALMA are also corroborated by data from Owens Valley Radio Observatory's Combined Array for Research in Milimeter-Wave Astronomy (CARMA), as reported by Stephens et al. (2014). In addition, and perhaps most importantly, the CARMA date show a magnetic field morphology of at 0.6 arcsecond resolution. The polarization vectors appear to show a magnetic field perpendicula to the disk orientation.

We now turn to jets associated with AGNs and quasars.

\section{Concurrent Variability of AGN Jet Sources as an Indication of Jet Launch or Jet-Cloud Interactions}

As examples, we now turn to two such observations in this paper, the concurrent radio and $\mathrm{x}$ ray variability of Centaurus A (Beall et al., 1978), and the $\gamma$-ray, UV, and IR concurrent variability discussed by Bonning et al., (2009) using the Fermi LAT and Swift spacecraft. 


\subsection{Radio and X-ray Variability Measurements of Centaurus A (NGC 5128)}

The first detection of concurrent, multifrequency variability of an AGN came from observations of Centaurus A (Beall et al., 1978, see Figure 1 of that paper). Beall et al. conducted the observing campaign of Cen $\mathrm{A}$ at three different radio frequencies in conjunction with observations from two different $\mathrm{x}$-ray instruments on the OSO-8 spacecraft in the 2-6 keV and $100 \mathrm{keV} x$-ray ranges. These data were obtained over a period of a few weeks, with the Stanford Interferometer at $10.7 \mathrm{GHz}$ obtaining the most data. Beall et al. also used data from other epochs to construct a decade-long radio and x-ray light curve of the source. Figure 1a of Beall (2011) shows the radio data during the interval of the OSO-8 x-ray observations, as well as the much longer timescale flaring behavior evident in the three different radio frequencies and at both low-energy (2-6 keV, see Figure 1b of Beall, 2011) and in high-energy ( $100 \mathrm{keV}$, see Figure 1c of Beall, 2011) x-rays. As noted by Beall (2011), a perusal of Figure 1a in that paper shows that the radio data (represented as a "+" in that figure) generally rise during 1973 to reach a peak in mid-1974, then decline to a relative minimum in mid-1975, only to go through a second peak toward the end of 1975, and a subsequent decline toward the end of 1976. This pattern of behavior is also shown in the $\sim 30 \mathrm{GHz}$ data and the $\sim 90 \mathrm{GHz}$ data albeit with less coverage at the higher two radio frequencies. These data are discussed in more detail in Besll (2015).

A plausible hypothesis for the Cen A observations is that they arise from physical processes associated with the "launch" of an astrophysical jet into the complex structures in the core of Centaurus A. The timescale of the evolution from early 1973 through 1977 appears to be associated with the evolution of a larger structure over a more extended region. Again, the observations are consistent with the interaction of an astrophysical jet with an interstellar cloud in the core of Cen A.

It is clear from this discussion that a distinction needs to be made about which observational signatures are associated with the jet launch, the jet itself, and the ambient medium's reaction to the jet. In considering such a scenario applied to microquasars, the observations of Sco X-1 by Fomalont, Geldzahler, and Bradshaw (2001), as discussed by Beall et al. (2013) are extremely informative. Such patterns of flaring have also been observed in other AGNs, including Blazars.

\subsection{A Jet Launch Coincident with the 3C454.3 Multi-frequency Flaring}

Bonning et al. (2009) performed an analysis of the multi-wavelength data from the blazar, 3C454.3, using IR and optical observations from the SMARTS telescopes, optical, UV and X-ray data from the Swift satellite, and public-release $\gamma$-ray data from the Fermi LAT experiment. In that work, she demonstrated an excellent correlation between the IR, optical, UV and $\gamma$-ray light curves, with a time lag of less than one day.

Urry (2011) noted that 3C454.3 can be a laboratory for multifrequency variability in Blazars. While a more precise analysis of the data will be required to determine the characteristics of the emitting regions for the observed concurrent flaring at the different frequencies, the pattern of a correlation between low-energy and higher-energy variability is consistent with that observed for Cen A, albeit with the proviso that the energetics of the radiating particles in $3 \mathrm{C} 454.3$ is considerably greater. 


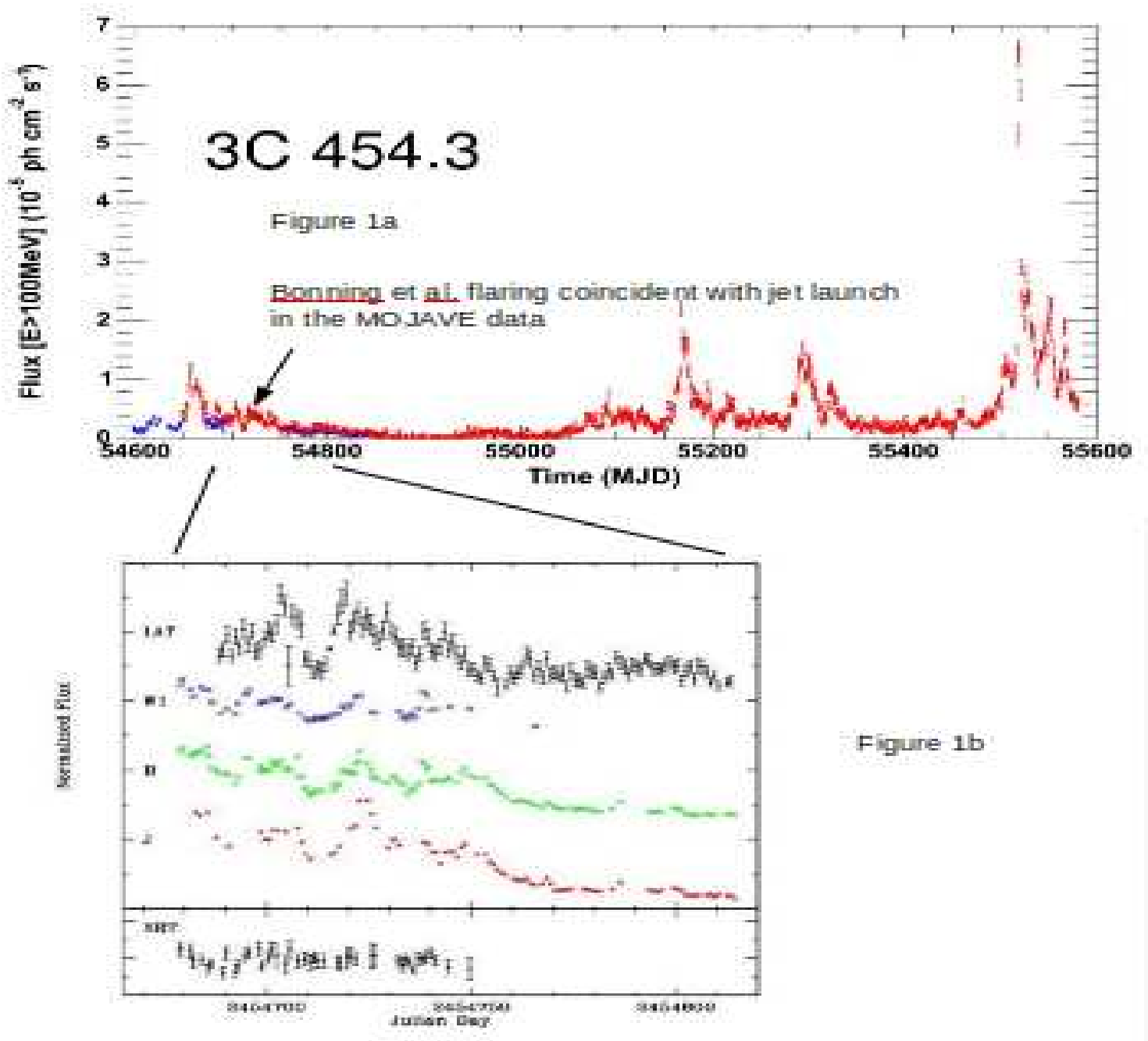

Figure 2: Fermi LAT data (see, e.g., Lott et al. 2013) showing the time history of the flaring from 3C454.3 (Figure 2a), along with the Bonning et al. (2009) data showing concurrent $\gamma$-ray, IR, optical, and UV data (Figure 2b). The time of the jet launch from the MOJAVE "movie" is illustrated by the arrow. A more complete discussion can be found in Beall (2015)

A perusal of the data for 3C454.3 at milliarcsecond scales taken from the MOJAVE VLBA campaign during the Bonning et al (2009) flare show that the time-dependent flare occurs during launch of a new component of the jet that originates from the core.

The pattern of variability reported by Bonning et al. is consistent with the injection of relativistic particles into a region with relatively high particle and radiation densities (i.e., an interstellar cloud). The picture that emerges, therefore, is consistent with the observations of spatially and temporally resolved galactic microquasars and AGN jets.

It should not escape our notice that other epochs in the 3C454.3 data from MOJAVE and Fermi 


\section{Additional MOJAVE jet launches}

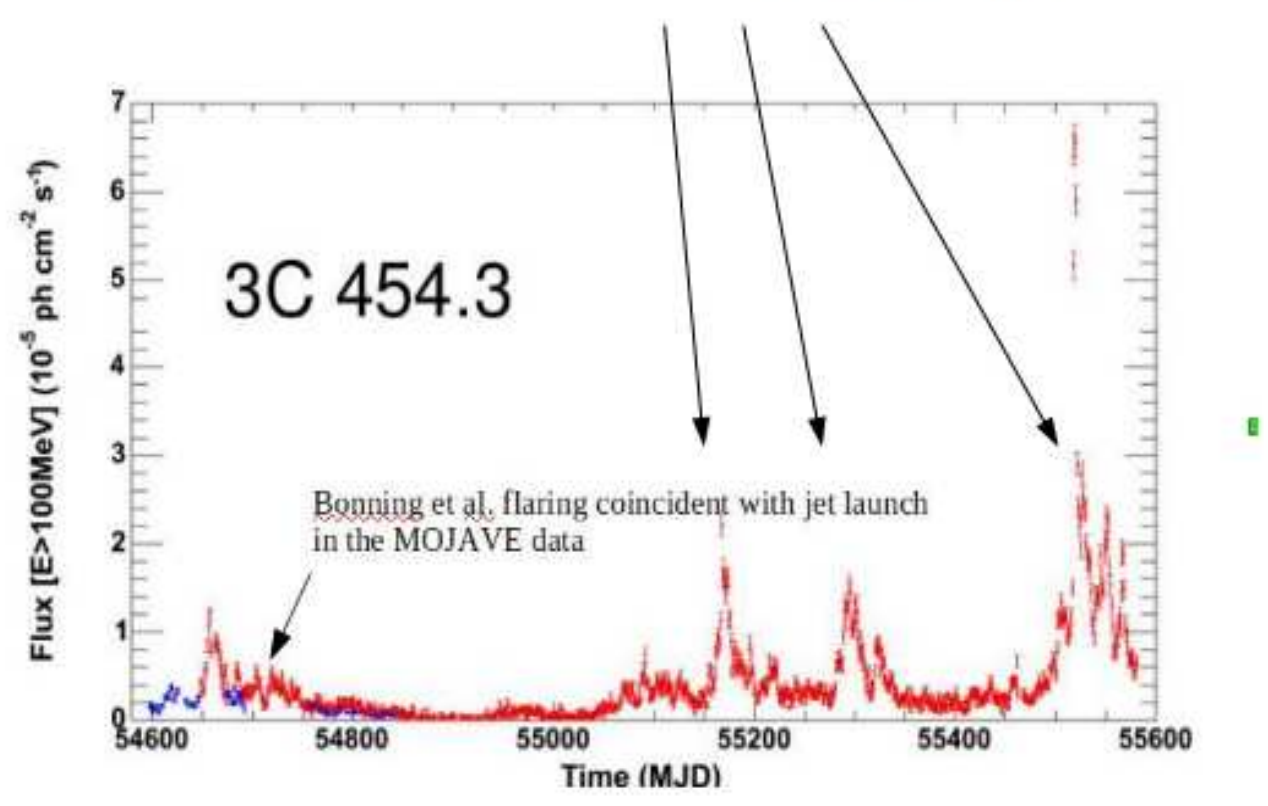

Figure 3: Fermi LAT data (see, e.g., Lott et al. 2013) showing the time history of the flaring from 3C454.3 along with times for several additional flares in the MOJAVE data sets, The flares are similar to those shown in Figure 2 above, and clearly indicate the correlation of $\gamma$-ray flares with the jet launches. These associations have also been pointed out by Jorstadt et al. 2013, who provide a detailed review of three outbursts in their paper. The direct correlation of the Fermi LAT and the MOJAVE data can clearly be seen in the figure. The three additional flares are similar in temporal structure to those illustrated in Figure 2. In total, the data presented here show four concurrent $\gamma$-ray and radio flares associated with the launch of material that comprises an astrophysical jets.

LAT also show similar periods of injection of radio blobs that are associated with a double-peaked structure in the $\gamma$-ray light. The Fermi LAT data (see, e.g., Lott et al. 2013) show that the time history of the flaring from $3 \mathrm{C} 454.3$ are associated with times for several additional flares in the MOJAVE data sets (see Figure 3). The flares are similar to those shown in Figure 2, and clearly indicate the correlation of $\gamma$-ray flares with the jet launches. These associations have also been pointed out by Jorstadt et al. 2013, who provide a detailed review of three outbursts in their paper. The direct correlation of the Fermi LAT and the MOJAVE data can clearly be seen in the figure. The data show evidence for the repeated launching of the astrophysical jets into the ambient medium. The jet structure is apparently not a continuous injection over long time scales, but an injection of a series of "blobs" that are co-aligned along some preferred axis.

This last statement cannot, however, be used as an argument for simple, spherically symmetric models of these sources. This is especially true since the injected material (itself unlikely to be symmetric by any definitions of the word) immediately interacts with highly asymmetric magnetic fields and structures in the accelerating region and in the ambient medium in the source's core. 
The result of these phenomena is a dynamic, highly anisotropic emitting region that produces anisotropic radiation over the entire electromagnetic spectrum.

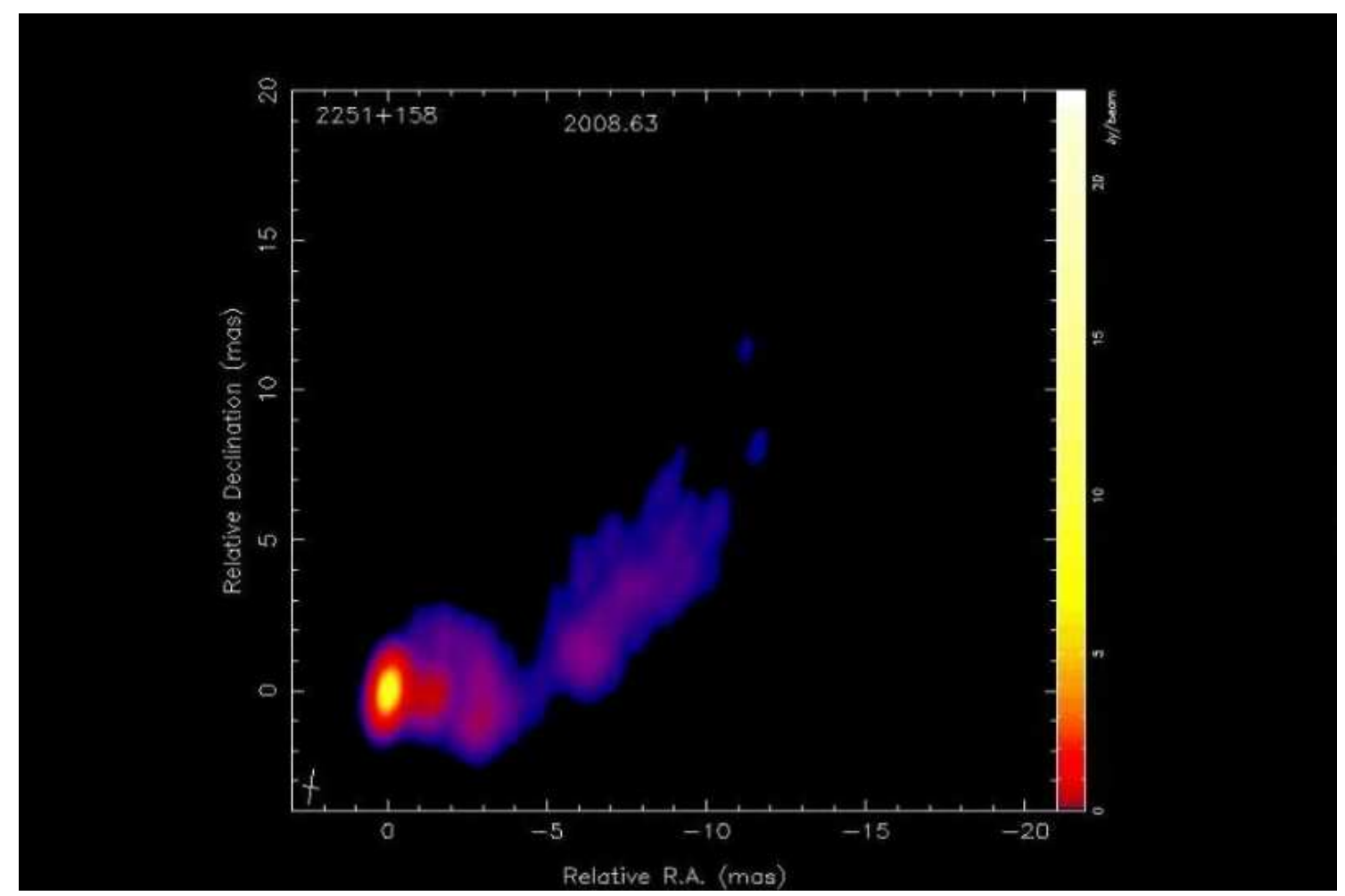

Figure 4: 3C454.3 shown at milliarcsecond scales for data taken from the MOJAVE VLBA campaign during the Bonning et al (2009) flare. A perusal of the time-dependent flare clearly shows that the jet launch from the core of the AGN is coincident with the double peaked flaring shown in the Bonning et al. results.

For the Cen A data, as for data from 3C454.3, it was the concurrent variability that suggests that the radio to x-ray (in Cen A's case) and the IR, optical, and UV to $\gamma$-ray fluxes (in 3C454.3's case) are created in the same region. This leads to the possibility of estimates of the source parameters that are obtained from models of these sources. VLBI observations of cores vs. jets (see, e.g., the study of BL Lac by Bach et al. 2006) show the structures of the core vs. jet as they change in frequency and time. It has thus become possible to separate and study the time variability of the jet and the core of AGN at remarkably fine temporal and spatial scales.

Van der Laan (1966) discussed the theoretical interpretation of cosmic radio data by assuming a source which contained uniform magnetic field, suffused with an isotropic distribution of relativistic electrons. The source, as it expanded, caused an evolution of the radio light curve at different frequencies. Each of the curves in Van der Laan's paper represents a factor of 2 difference in frequency, the vertical axis representing intensity of the radio flux and the horizontal axis representing an expansion timescale for the emitting region. Van der Laan's calculations show a marked difference between the peaks at various frequencies.

In the example of Cen A, the radio frequency bands track one another throughout the entire interval reported by Beall et al. (1978). The data from Cen A (as discussed more fully in Beall $(2008,2010)$ are, therefore, not consistent with van der Laan expansion (Van der Laan, 1966), since 
for van der Laan expansion, we would expect the different frequencies to achieve their maxima at different times. Even if the $10.7 \mathrm{GHz}$ data are in the synchrotron self-absorbed portion of the spectrum, as appears likely, there is no straightforward way to explain the behavior of the radio data via the van der Laan mechanism.

The observations in the hard x-ray at $100 \mathrm{keV}$ are also problematic, since the slope of the power-law spectrum at $100 \mathrm{keV}$ hardens (i.e., flattens) at one epoch in the observations. The hard $\mathrm{x}$-ray spectrum is unabsorbed, and therefore represents the spectrum of the underlying radiating electrons. Therefore, the most likely explanation for the changes in the spectrum of the Cen A data at $100 \mathrm{keV}$ (Beall, et al., 1978) is that the emitting region suffered an injection or reacceleration of energetic electrons.

A similar scenario can explain the reported radio variability. That is, the jet-ambient-medium interaction injected a population of energetic particles into a putative van der Laan "blob," or, equivalently, that there was a re-acceleration of the emitting electrons on a timescale short compared to the expansion time of the source.

An analysis of the $3 \mathrm{C} 120$ results compared with the data from the galactic microquasar, Sco X-1, undertaken by Beall (2006) shows a similar radio evolution, with rapidly moving "bullets" interacting with slower moving, expanding blobs. It is highly likely that the elements of these sources that are consistent with van der Laan expansion are the slower-moving, expanding blobs. I believe that the relativistically moving bullets, when they interact with these slower-moving blobs, are the genesis of the flaring that we see that seems like a re-acceleration of the emitting, relativistic particles. I note that a similar scenario could be operating in Cen A and 3C454.3.

This is not to say that the "slower-moving blobs" are not themselves moving relativistically. It is clear that the bi-polar lobes have significant enhancements in brightness due to relativistic Doppler boosting for the blobs moving toward us.

The true test of this hypothesis will require concurrent, multifrequency observations with resolutions sufficient to distinguish jet components from core emissions in galactic microquasars as well as for AGN jets.

One of the most remarkable sagas regarding the discovery of quasar-like activity in galactic sources comes from the decades long-investigation of Sco X-1 by Ed Fomalont, Barry Geldzahler, and Charlie Bradshaw (Fomalont, Geldzahler, and Bradshaw, et al. 2001). During their observations, an extended source changed relative position with respect to the primary object, disappeared, and then reappeared many times. We now know that they were observing a highly variable jet from a binary, neutron star system. The determinant observation was conducted using the Very Large Array (VLA) in Socorro, New Mexico and the VLBA interferometer (see, e.g., Beall, 2008) for a more complete discussion).

The observations of the concurrent IR, Optical, UV, and $\gamma$-ray variability of $3 \mathrm{C} 454.3$, and its associated jet launch in the MOJAVE data, argue for a reinvestigation of these data sets in the near future.

It is worthy of note that the milliarcsecond observations show a complex evolution of structure at parsec scales, including an apparently sharp change of directions associated with changes in the polarization of the radio light at that point in the jet's evolution (see Figure 2). Furthermore, the multi-frequency flares reported by Bonning et al. (2009) are consistent with the launch of another component of the astrophysical jet in the core region. 
Regarding the acceleration region and the possible mechanisms for the collimation of the jets, a number of models have been proposed (see, e.g., Kundt and Gopal-Krishna (2004), BisnovatyiKogan et al. (2002), Romanova and Lovelace (2009), and Bisnovatyi-Kogan, et al. (2013) that might help explain the complexity present in these data.

\section{Neutrino Emission from an Astrophysical Source}

I present in this section very recent results, published by the ICECUBE Colaboration (see, e.g., Science, $13 \mathrm{Jul}$ 2018), that were in preparation at the time of the Mondello Workshop but not yet publically available.

On 22 September 2017, IceCube detected a neutrino of very high energy, indicating that the particle might have originated from an AGN. The neutrino detectioin alert was promptly disseminated to other observing teams, and the Fermi-LAT reported that the direction of the neutrino was in line with a known gamma-ray source in an active state: the blazar TXS 0506+056. In addition, the MAGIC telescopes showed that a source of extremely high energies in the same region of the sky.

These detections suggest strongly that the contents of astrophysical jets from Blazars are hadronic. Clearly, more observations and modeling are necessary for this conclusion to be fully understood.

\section{Concluding Remarks}

The data discussed herein suggest a model for the jet structures in which beams or blobs of energetic plasmas propagate outward from the central engine to interact with the ambient medium in the central rgions of these sources. This ambient medium in many cases comes from prior ejecta from the central source, but can also come from clouds in the Broad Line Region. The jet can apparently also excavate large regions, as is suggested by the complex structures in, for example, 3C120. The physical processes which can accelerate and entrain the ambient medium through which the jet propagates have been discussed in detail in several venues (see, e.g., Rose et al., 1984, 1987, Beall, 1990, Beall et al., 2003, and Beall, 2010). It is also possible given analysies by Kundt and Gopal-Krishna (2004) and subsequent simulations, that the jets can consist of electronpositron pairs. This thesis is especially intriguing for pulsar-driven jets.

In this review, we have focused on the patterns of concurrent radio and x-ray variability for Cen A, and on concurrent radio, $\gamma$-ray, optical, IR, and UV variability for 3C454.3. The data can be interpreted as being associated with a jet-launch scenario for these sources, and suggests an association between a jet launch in the MOJAVE data for 3C454.3 and its $\gamma$-ray flare from Fermi LAT. In addition, jets from microquasars show similar patterns of variability to those of AGNs. These data require us to abandon our assumptions both of spherical symmetry and of single-zone productions in our models of these sources.

Clearly, the very recent results of neutrino emission associated with a single Blazar bears further study.

The author gratefully acknowledges the support of the Chief of Naval Research for these efforts. This research has made use of data from the MOJAVE database that is maintained by the 
MOJAVE team (Lister et al., 2009, AJ, 137, 3718). The author gratefully acknowledges the data provided by the MOJAVE Team for this paper.

\section{References}

[1] Fermi LAT Collaboration; Abdo, A. A.; Ackermann, M.; Ajello, M.; Axelsson, M.; Baldini, L.; Ballet, J.; Barbiellini, G.; Bastieri, D.; Baughman, B. M.; and 167 coauthors: 2009, Sciience, 326.1512F (Modulated High-Energy Gamma-Ray Emission from the Microquasar Cygnus X-3)

[2] Abdo, A. A.; Ackermann, M.; Ajello, M.; Atwood, W. B.; Axelsson, M.; Baldini, L.; Ballet, J.; Barbiellini, G.; Bastieri, D.; Baughman, B. M.; and 173 coauthors: 2009, Ap. J., 701L,123A (Fermi LAT Observations of LS I +61 Âř303: First Detection of an Orbital Modulation in GeV Gamma Rays)

[3] The Pierre Auger Collaboration, et al., 2007, Science 318, 938.

[4] Bach, U., Villata, M., Raiteri, C. M., et al. 2006, "Structure a variability in the VLBI jet of BL Lacertae during the WEBT campaigns (1995-2004)," A\&A, 456, 105.

[5] Beall, J.H. et al., 1978, Ap. J., 219, 836.

[6] Beall, J.H., Rose, W.K., 1980, Ap. J., 238, 579.

[7] Beall, J.H., 1987, Ap. J., 316, 227.

[8] Beall, J.H., 1990, Physical Processes in Hot Cosmic Plasmas (Kluwer: Dordrecht), W. Brinkman, A. C. Fabian, \& F. Giovannelli, eds., pp. 341-355.

[9] Beall, J.H., Bednarek, W., 2002, Ap. J., 569, 343.

[10] Beall, J.H., 2002, in Multifrequency Behaviour of High Energy Cosmic Sources, F. Giovannelli \& L. Sabau-Graziati (eds.), Mem. S.A.It. 73, 379.

[11] Beall, J.H., 2003, in Multifrequency Behavior of High Energy Cosmic Sources, Chin. J. Astron. Astrophys. 3, Suppl., 373.

[12] Beall, J.H., 2009, Societa Italiana di Fisica, 98, 283-294.

[13] Beall, J.H., 2010, in Multifrequency Behaviour of High Energy Cosmic Sources, Frascati Workship 2009, F. Giovannelli \& L. Sabau-Graziati (eds.), Mem. S.A.It. 81, 395.

[14] Beall, J.H., et al., 2013, Acta Polytechnica, 53 (Supplement), 683-686.

[15] Beall, J.H., 2011, Mem. S. A. It., 83, 283-290.

[16] Beall, J.H., 2015, Mem. S. A. It., 83, 283-290.

[17] Benford, Gregory, and Protheroe, R.J., 2008, M.N.R.A.S., A383, 417âĂŞ816.

[18] Bisnovatyi-Kogan, Genaddi, Ardelyan, Nikolai, and Moiseenko, Sergei, 2002, Mem. S.A.It. 73, 1134., in Proceedings of the Vulcano Workshop on High-Energy Cosmic Sources, 2001

[19] Bisnovatyi-Kogan, Genaddi, Klepnev, Alexandr S., and Lovelace, Richard V.E., 2013, Acta Polytechnica, 53 (Supplement), 667-682.

[20] Bonning, E. W., Bailyn, C., Urry, C. M., Buxton, M., Fossati, G., Maraschi, L., Coppi, P.,Scalzo, R., Isler, J., Kaptur, A. 2009, Astrophys. J. Lett., 697, L81.

[21] Carilli, C.L. et al.: 1996, The Astron Astrophys Rev 7, 1â̆̆Ş54 (Cyg A). 
[22] Chaty, S.: 1998, PhD Thesis (Multi-wavelength study of the microquasar GRS 1915+105 and of high-energy binary sources in the Galaxy)

[23] Chaty, S., 2007, in Frontier Objects in Astrophysics and Particle Physics, F. Giovannelli \& G. Mannocchi (eds.), Italian Physical Society, Editrice Compositori, Bologna, Italy, 93, 329.

[24] Cooper et al., 2007, Ap.J. Supplement, 171, 376.

[25] Dar, Arnon, 2009, private communication.

[26] Doolin, S., and Blundell, K.M., 2009, Ap. J.,698, L23.

[27] Fomalont, E., Geldzahler, B., Bradshaw, C., 2001, Ap. J. 558, 283-301.

[28] Giovannelli, F., Sabau-Graziati, L., 2004, Space Science Reviews, 112, 1-443 (Kluwer Academic Publishers: Netherlands).

[29] Gómez et al. 2000, Science 289, 2317.

[30] Hiribayashi et al., 2000 PASJ 52, 997.

[31] Hannikainen, D.C., Rodriguez, J., 2008, in Multifrequency Behavior of High Energy Cosmic Sources, Chin. J. Astron. Astrophys. 8, Suppl., 341.

[32] Hawley, J.F., 2003, Phys. Plasmas 10, 1946/

[33] Jorstad, S.G., Marscher, A.P., Lister, M.L., Stirling, A.M., Cawthorne, T.V. et al., 2005, Astron. J. 130, 1418-1465.

[34] Jorstadt, S., Marscher, A., Stevens, J., Smith, P., Forster, J. et al., 2006, in Multifrequency Behavior of High Energy Cosmic Sources, Chin. J. Astron. Astrophys. 6, Suppl. 1, 247.

[35] Jorstad, Svetlana G., et al., 2013, Astrophysical Journal, 773, 147.

[36] Kundt, W. and Gopal-Krishna, 2004, Journal of Astrophysics and Astronomy, 25, 115-127.

[37] Krause, M., Camenzind, M., 2003, in The Physics of Relativistic Jets in the CHANDRA and XMM Era, New Astron. Rev. 47, 573.

[38] Lightman, A. P., Eardley, D. N., 1974, Ap. J. Letters 187, L1.

[39] Lister et al., 2009, A.J., 137, 3718.

[40] Madejski, Greg M., et al., 1999, ApJ, 521, 145-154.

[41] Marscher, A.P., et al., 2002, Nature, 417, 625-627.

[42] Marscher, A.P., 2006, in Multifrequency Behavior of High Energy Cosmic Sources, Chin. J. Astron. Astrophys. 6, Suppl. 1, 262.

[43] Mioduszewski, A. J., Rupen, M. P., Walker, R. C., Schillemat, K. M., \& Taylor, G. B. 2004, BAAS, $36,967$.

[44] Mirabel, I. F.; Dhawan, V.; Chaty, S.; Rodriguez, L. F.; Marti, J.; Robinson, C. R.; Swank, J.; Geballe, T., 1998, A.\& A., 330L, 9M (Accretion instabilities and jet formation in GRS 1915+105).

[45] Mirabel, I. F.; Chaty, S.; Rodriguez, L. F.; Sauvage, M., 2015, "Jet-induced star formation by a microquasar", in Extragalactic jets from every angle, Proceedings of the International Astronomical Union, IAU Symposium, Volume 313, pp. 370-373.

[46] Mushotzky, R.F., Serlemitsos, P.J., Becker, R.H., Boldt, E.A., and Holt, S.S., Ap. J. 220, 790-797. 
[47] ICECUBE COLLABORATION, Science 13 Jul 2018: Vol. 361, Issue 6398, pp. 147-151.

[48] Paredes, J., 2007, in Frontier Objects in Astrophysics and Particle Physics, F. Giovannelli \& G. Mannocchi (eds.), Italian Physical Society, Editrice Compositori, Bologna, Italy, 93, 341.

[49] Rahoui, F.; Chaty, S.; Rodriguez, J.; Fuchs, Y.; Mirabel, F., 2008, "Proceedings of the VII Microquasar Workshop: Microquasars and Beyond. September 1 - 5, 2008. Foca, Izmir, Turkey. Published online at http://pos.sissa.it/cgi-bin/reader/conf.cgi?confid=62, p.36" (Multi-wavelength monitoring of the microquasar GRS 1915+105)

[50] Romanova, M. and Lovelace, R., 2005, 2009, Triggering of Relativistic Jets, (Instituto de Astronomia, Universidad Nacional Autonoma de Mexico, William H. Lee and Enrico Ramirez-Ruiz, eds.); also at arXiv:0901.4753v1, astro-ph.HE, 29 Jan 2009.

[51] Rose, W.K., et al., 1984, Ap. J. 280, 550.

[52] Rose, W.K., et al., 1987, Ap. J. 314, 95.

[53] Ulrich, Marie-Helene, Maraschi, Laura, and Urry, C. Megan, 1997, Ann. Rev. Astron. Astrophys, 35, 445-502.

[54] Urry, C. Megan, 2011, J. Astrophys. Astr. 32, 139âĂŞ145

[55] van der Laan, H., 1966, Nature 211, 1131.

[56] Zanni, C., Murante, G., Bodo, G., Massaglia, S., Rossi, P., Ferrari, A., 2005 Astron. Astrophys., 429, 399.

\section{DISCUSSION}

MARTIN TOPINKA QUESTION: You showed jet bending. Taking into account the microquasar, quasar, and GRB analogs for jet physics, do you expect any observational piece of evidence to see similar bending in the late GRB afterglows?

JIM BEALL ANSWER: I believe we will see the effects of jet bending in GRB afterglows, although these might be interpreted as a "halo" of emission around the source. It is likely that experiments capable of detecting polarization in radio light could be the principal indicia of this process.

ANDREJ ZDIARSKI QUESTION: Two questions: First, you talked about blobs. But there are two kinds of jets. Some are extended, also. Second, the presence of protons in jets does not impy hadronic processes producing neutrinos.

JIM BEALL ANSWER: Good points. Regarding your first comment, I agree that there are continuous jets, usually associated with pulsars, as well as those which evolve in "blobs" that seem to come from an episodic emission of the jets. The jets with blobs are clearly also continuous structures. in my own view is that these are associated with instabilities from the inner regions of accretion disks around compact objects. Clearly, the jets originating from accretion disk can be of a different character than those originating from pulsars. Regarding your second question, I also agree that the presence of hadrons in jets does not necessarily demand the production of neutrinos, but requires a certain threshold energy.

NOTE ADDED IN PROOF: The recent neutrino detection from a flaring Blazar suggests that the energetic criterion is met in at least that source, and therefore Blazars can be significant sources of neutrinos. 